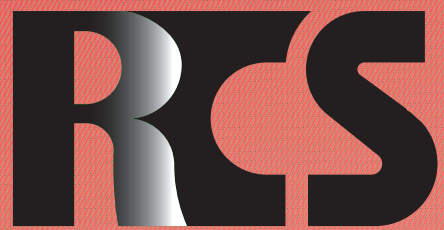

Depósito legal ppi $201502 Z U 4662$

Esta publicación científica en formato digital es continuidad de la revista impresa Depósito Legal: pp $197402 Z U 789$

- ISSN: 1315-9518 • ISSN-E: 2477-9431

Revista de Ciencias Sociales

Universidad del Zulia. Revista de la Facultad de Ciencias Económicas y Sociales Vol. XXVII. No. 2

Abril-Junio 2021

Esta publicación científica en formato digital es continuidad de la revista impresa Depósito Legal: pp $197402 Z$ Z789 ISSN: 1315-9518 


\title{
Estrategias pedagógicas para el aprendizaje y desarrollo de competencias investigativas en estudiantes universitarios
}

\author{
Hernández Sánchez, Irmina Beatriz* \\ Lay, Nelson** \\ Herrera, Henry $^{* * *}$ \\ Rodríguez, Marcela ${ }^{* * * *}$
}

\section{Resumen}

Los nuevos retos de la educación superior demandan la adquisición y desarrollo de competencias investigativas que puedan ser útiles para responder a las nuevas exigencias del entorno laboral, lo cual, es un fuerte motivador para replantear las estrategias pedagógicas empleadas, dado que se requieren nuevas formas de lograr el conocimiento en los estudiantes, integrando metodologías basadas en el trabajo en equipo, como es el caso del aprendizaje cooperativo. El objetivo de este artículo es describir la experiencia pedagógica de la implementación de un proyecto de aula basado en el aprendizaje cooperativo para el desarrollo de competencias investigativas psicométricas en estudiantes universitarios, a partir del análisis de la confiabilidad y validez de instrumentos de medición y evaluación psicológica. Se encontró que este tipo de estrategias favorece al desarrollo de habilidades para la investigación científica, potencia el trabajo en equipo y aumenta las interacciones sociales entre el alumnado. Se concluye, que el aprendizaje cooperativo es efectivo para la formación de diferentes competencias investigativas, así como otras habilidades importantes para acceder a oportunidades laborales actuales o futuras donde se requieran este tipo competencias. Se recomienda implementar este tipo de estrategias para lograr mejores resultados de aprendizaje en los estudiantes universitarios.

Palabras clave: Estrategias pedagógicas; aprendizaje cooperativo; formación por competencias; competencias investigativas; psicometría.

\footnotetext{
Magister en Psicología. Docente de la Universidad de la Costa, Colombia. E-mail: ihernand8@ecuc.edu.co iD ORCID: https://orcid.org/0000-0002-7024-2668

** Doctor en Sociología. Docente de la Facultad de Educación y Ciencias Sociales de la Universidad Andres Bello, Chile, Chile. E-mail: nelson.lay@unab.cl (iD) ORCID: https://orcid.org/0000-0001-8501-7570

*** Magister en Estadística. Docente de la Universidad de la Costa, Colombia. E-mail: hherrera8@cuc.edu.co iD ORCID: https://orcid.org/0000-0001-5352-1537

***** Magister en Administración de Empresas. Docente de la Universidad Andrés Bello, Chile. E-mail: marce.borbaran@a hotmail.com (D) ORCID: https://orcid.org/0000-0001-6131-1855
} 


\title{
Pedagogical strategies for the learning and development of research competences in university students
}

\begin{abstract}
The new challenges of higher education demand the acquisition and development of investigative competences that may be useful to respond to the new demands of the work environment, which is a strong motivator to rethink the pedagogical strategies used, since new forms of teaching are required. achieve knowledge in students, integrating methodologies based on teamwork, as is the case of cooperative learning. The objective of this article is to describe the pedagogical experience of the implementation of a classroom project based on cooperative learning for the development of psychometric investigative competences in university students, based on the analysis of the reliability and validity of psychological measurement and evaluation instruments. It was found that this type of strategies favors the development of skills for scientific research, enhances teamwork and increases social interactions among students. It is concluded that cooperative learning is effective for the formation of different investigative competencies, as well as other important skills to access current or future job opportunities where this type of competency is required. It is recommended to implement this type of strategies to achieve better learning results in university students.
\end{abstract}

Keywords: Pedagogical strategies; cooperative learning; training by competencies; investigative competences; psychometrics.

\section{Introducción}

La educación superior actual está marcada por diferentes retos, muchos de estos están orientados al desarrollo de competencias académicas relacionadas con la investigación científica, que no solo son útiles para el óptimo desarrollo de varias de las actividades propuestas en las clases, sino también para contribuir a la producción científica desde la academia (Niebles, Martínez-Bustos y Niebles-Núñez, 2020).

Considerando que una de las metas del desarrollo sostenible enmarcado en la educación de calidad pretende lograr para el 2030 un aumento considerable del número de jóvenes y adultos que tienen las competencias necesarias, en particular técnicas y profesionales, para acceder al empleo, el trabajo decente y el emprendimiento (Naciones Unidas, 2018), se hace necesario que los docentes incorporen dentro de su plan de formación estrategias de pedagogías que permitan a los estudiantes el desarrollo de competencias que doten a los futuros profesionales de conocimientos y destrezas importantes para acceder a mejores oportunidades de empleo y a la posibilidad de estudios más avanzados donde se requiera contar con dichas competencias, haciendo especial énfasis a las competencias direccionadas a la investigación (Lay, Ramírez y Parra, 2019; Cejas, et al., 2019).

En este sentido, es importante que el acto pedagógico se acompañe de un trabajo en equipo donde profesores y estudiantes articulen sus conocimientos así como sus aportes, asumiendo los primeros el papel de guía, supervisor y dinamizador del proceso de aprendizaje de los estudiantes, mientras que a los segundos, se les exija un rol más activo y participativo dentro y fuera del aula (González, García y Ramírez, 2015). Es decir, se hace necesaria la inclusión e implementación de estrategias de enseñanza-aprendizaje con base en el trabajo cooperativo, teniendo en cuenta que este representa un medio idóneo para fomentar un aprendizaje por competencias, incorporando tareas orientadas a formar 
personas y profesionales con capacidad de responder a las necesidades de la sociedad (Cifuentes y Meseguer, 2015; Compte y Sánchez, 2019; Martínez-Garcés y GarcésFuenmayor, 2020).

Por lo anterior, esta experiencia educativa pretende mostrar los resultados obtenidos de la implementación de una estrategia de aprendizaje cooperativo para el desarrollo de competencias de medición, evaluación y análisis psicométrico, como parte de un proyecto de aula de la asignatura fundamentos de medición y evaluación, del programa de psicología de una institución de educación superior de la ciudad de Barranquilla (Colombia), con el propósito de establecer la confiabilidad y validez de instrumentos de medición utilizados para medir las variables: Comportamiento organizacional ciudadano, autonomía, clima escolar y habilidades sociales a partir del análisis estadístico de las bases de datos derivadas de la aplicación previa de estos instrumentos.

\section{Fundamentación teórica}

\subsection{El aprendizaje: Desde la pirámide del aprendizaje hasta el aprendizaje cooperativo}

El aprendizaje, es un proceso a través del cual se consigue un cambio en los conocimientos, las creencias, la conducta y las actitudes; que se deben al resultado de experiencias vividas que incrementan el potencial y capacidades para desempeñarse de mejor manera en las actividades presentes y futuras (Ambrose, et al., 2017). Es decir, es un proceso dinámico con el fin de lograr un cambio en la conducta y en los procesos cognitivos, como consecuencia de la experiencia que se obtiene de la adaptación a ambientes cambiantes y de exigencia (Esguerra y Guerrero, 2010; Garcés y Mora, 2020).

Para que el aprendizaje ocurra, es necesario que el estudiante desarrolle o tenga la capacidad para establecer relaciones, de manera no arbitraria y sustancial, entre la nueva información adquirida con los conocimientos y experiencias previas así como familiares, que constituyen su estructura de conocimientos, además debe tener la disposición de aprender y darle significado a lo que aprende, así como contar con los materiales y contenidos de aprendizaje necesarios para potenciar y darle significado a dichos conocimientos (Castillo, Yahuita y Garabito, 2006), de allí que el docente y sus estrategias de enseñanza, juegan un papel importante en la adquisición y desarrollo de esos nuevos saberes.

Considerando lo expuesto por Valbuena (2017); y, Gómez-Ejerique y López-Cantos (2019), acerca de la pirámide del aprendizaje construida por Cody Blair (ver Figura I), es posible afirmar que, para lograr la adquisición de conocimientos o saberes, es decir, el aprendizaje, es posible utilizar diferentes estrategias, cada una de las cuales genera un porcentaje de aprendizaje, lo cual podría indicar cuales son las estrategias más efectivas para lograr un aprendizaje más profundo. 


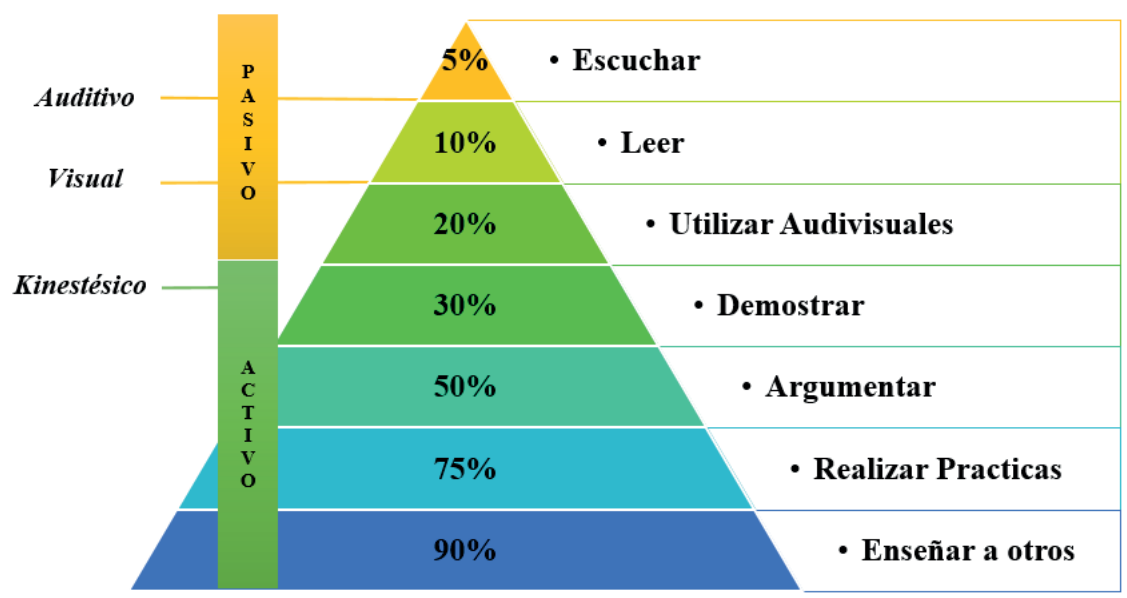

Fuente: Elaboración propia, 2020.

Figura I: La pirámide del aprendizaje de Cody Blair

En este sentido, como se aprecia en la Figura I, por medio de estrategias magistrales como escuchar una clase, tan solo se logra un $5 \%$ del aprendizaje en los estudiantes, entre tanto, se consigue un $10 \%$ a través de la lectura de libros u otros materiales bibliográficos, mientras que, con el uso de recursos audiovisuales como videoconferencias solo se adquiere un $20 \%$ de conocimientos. Este tipo de estrategias son consideradas como pasivas, de tipo auditivo y visual, teniendo en cuenta que el estudiante asume solamente el rol de receptor del conocimiento y no de ejecutor de este.

De igual manera, se encuentran estrategias donde el estudiante asume un rol más activo y autogenerador del conocimiento, las cuales son de tipo kinestésicas, es decir, relacionadas con el hacer, dentro de las que encuentran las exposiciones o exhibiciones correspondientes a estrategias de demostración, con las que se logra un $30 \%$ del aprendizaje, en este grupo también se encuentran los debates u otros métodos de argumentación por parte del estudiante, con las cuales se consigue un $50 \%$ de los conocimientos.

Por último, se encuentran estrategias como proyectos de aula o actividades en laboratorios, donde el estudiante aprende extrapolando la teoría a la práctica, con la cual se consigue un $75 \%$ del aprendizaje, y finalmente, se logra un $90 \%$ del aprendizaje si se comparten los conocimientos adquiridos con otros, es decir, si se enseña a otros aquello que ya se sabe.

Por otra parte, para abordar el aprendizaje cooperativo, se debe tener en cuenta que cooperar, consiste en trabajar en equipo con el propósito de lograr objetivos en común, el aprendizaje colaborativo podría definirse como un conjunto de métodos de instrucción y trabajo en conjunto, entre estudiantes generalmente heterogéneos en rendimiento, cada uno de los cuales es responsable no solo de aprender por sí mismo, sino también de ayudar a que los demás integrantes también aprendan para conseguir una meta compartida que se han fijado como grupo, lo cual favorece 
a la valoración positiva del trabajo académico personal y la motivación para aprender así como enseñar a otros, promoviendo la interacción entre ellos y la integración de sus distintos puntos de vista en función de lograr dicha meta (López y Acuña, 2015).

De acuerdo con Vallet-Bellmunt, et al. (2017), el aprendizaje cooperativo combina la teoría, la investigación y la práctica, representando un procedimiento poderoso de aprendizaje, donde docentes y estudiantes trabajan en conjunto para lograr los objetivos de la clase. Respecto a la relación interpersonal que se establece entre el profesor y el estudiante en el aprendizaje cooperativo, Dejo-Oricain (2015) asegura que:

Mientras los grupos trabajan sobre los contenidos propuestos, el profesor se mueve por la clase prestando atención al trabajo de los distintos grupos, con objeto de comprobar que la interacción es la adecuada. También interviene en los equipos para controlar el desarrollo del trabajo, realizar correcciones y ofrecer los apoyos necesarios, asegurándose de que todos los grupos cuenten con el material necesario para realizar las actividades. Junto a esta relación que se mantiene en el aula, se establecen horarios de tutoría tanto individualizadas como en grupos pequeños fuera del aula. Esta labor de tutoría tiene consecuencias altamente positivas. Por un lado, se observa una mayor satisfacción del estudiante y una mejor actitud hacia la asignatura. Por otro lado, también mejora la percepción que el profesor tiene de los estudiantes. (p. 345)

En resumen, la relación profesorestudiante se caracteriza por un trabajo de orientación, tutoría y acompañamiento por parte del profesor hacia el trabajo en los estudiantes, mientras que los estudiantes, se comprometen entre sí para desarrollar los contenidos propuestos, realizar las correcciones señaladas por el docente y asistir a las tutorías para darle una mejor forma a su trabajo, despejar dudas y complementar lo que están realizando; lo anterior, no solo permite una mejor realización del trabajo, sino un mejor desarrollo de competencias y una relación más cercana y productiva entre ambas partes durante el proceso de enseñanzaaprendizaje.

\subsection{Estratégicas pedagógicas}

Según Gamboa, García y Beltrán (2013); junto a Parra, et al. (2018), al hablar de estrategias pedagógicas se refieren a todas aquellas acciones llevadas a cabo por el docente con el propósito de facilitar el proceso de formación y aprendizaje de los estudiantes; las cuales en la actualidad deben estar ajustadas al contexto, a las necesidades e intereses de los estudiantes, a la misión y visión institucional y a las demandas de una sociedad globalizado y tecnológicamente avanzada (Lucumi y González, 2015; Coaquira, 2020), permitiendo el desarrollo de competencias necesarias para desenvolverse exitosamente durante su formación profesional y posteriormente en el contexto laboral.

Existen diferentes tipos de estrategias pedagógicas que dinamizan y hacen más variado el proceso de aprendizaje de los estudiantes. Algunas de estas estrategias se presentación a continuación en la Figura II. 


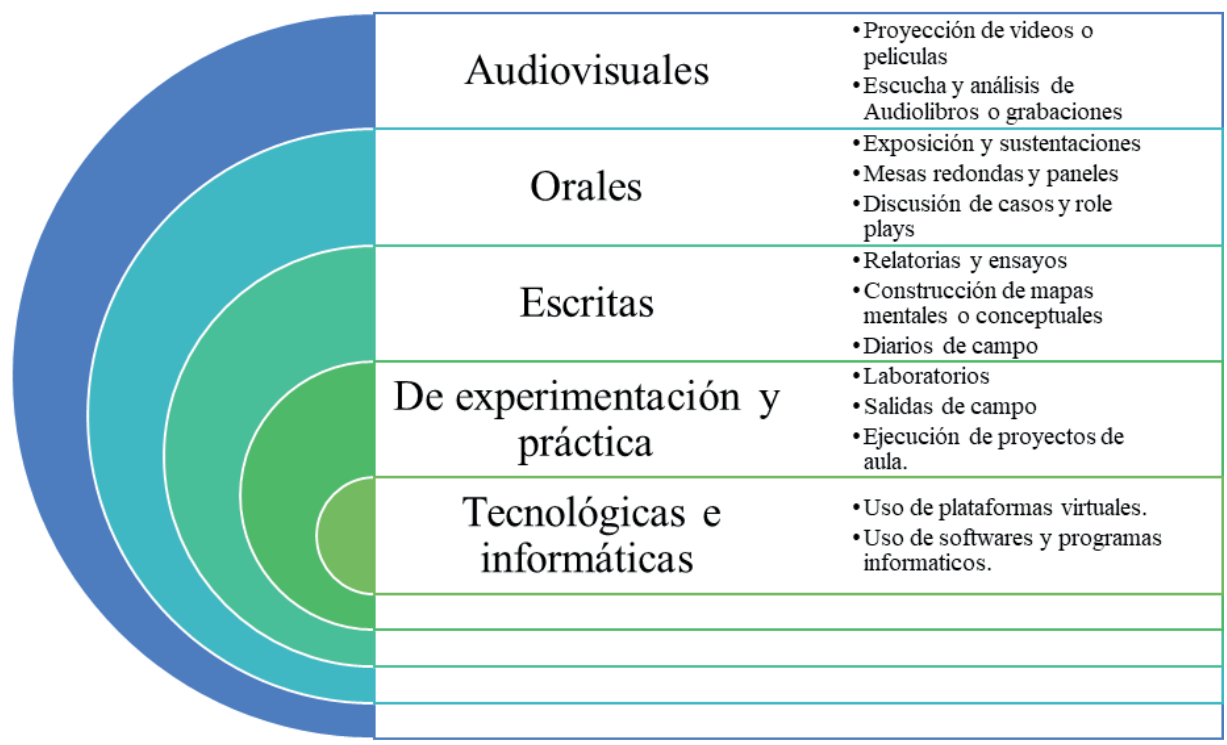

Fuente: Elaboración propia, 2020.

Figura II: Estrategias pedagógicas

\subsection{Desarrollo y formación de competencias: Hacia la investigación científica}

García, Tejeda y Torres (2014), definen la competencia como un conjunto de conocimientos, habilidades, destrezas $\mathrm{y}$ actitudes que capacitan y movilizan a una persona de forma íntegra, para actuar eficazmente ante las demandas de un contexto determinado. En este sentido, una formación basada en el desarrollo de competencias implica un proceso de enseñanza-aprendizaje que pretende facilitar la transmisión de conocimientos y la generación de habilidades y destrezas, desarrollando en el individuo la capacidad para poner en práctica dichas competencias adquiridas, con el fin de dar solución a situaciones emergentes que puedan ocurrir en los diferentes contextos de su realidad (Vargas, Casanova y Montanaro, 2001; Rabanal, et al., 2020).
En este nuevo proceso, el aprendizaje del alumno es lo más importante, más allá que la enseñanza del profesor, de allí que este asuma el protagonismo en su proceso formativo, gestionando su propio aprendizaje con el apoyo, asesoría y orientación del docente (Martínez, Martínez y Muñoz-Cantero, 2008; Pirela, et al., 2019). Este enfoque en el contexto de la educación superior favorece el refuerzo de competencias genéricas, pero fundamentalmente permite el desarrollo de nuevas competencias más específicas, entre ellas la formación investigativa (Rosario y Ferrer, 2014; Barreiro, 2015).

De este modo, las competencias de investigación científica pueden entenderse como "la capacidad de emplear los conocimientos científicos de un individuo y al uso de ese conocimiento para identificar problemas, adquirir nuevos conocimientos, explicar fenómenos científicos y extraer conclusiones basadas en pruebas sobre 
cuestiones relacionadas con la ciencia" (Organización para la Cooperación y el Desarrollo Económico [OCDE], 2006, p.13), es decir, implican la apropiación y uso de los conocimientos científicos que posee una persona para identificar, comprender, interpretar y explicar fenómenos de su realidad bajo el marco de la ciencia, para llegar a conclusiones científicas.

Según Rubio, et al. (2018), las competencias investigativas en los diferentes profesionales de las distintas disciplinas, favorecen al desarrollo social, a la innovación y la competitividad empresarial, de ahí que cada vez más se requieran profesionales que desde la academia, se formen en este tipo de competencias, considerando que en este tercer milenio, caracterizado especialmente por un extraordinario y vertiginoso avance científico, se necesita formar a los futuros profesionales en competencias científicas investigativas, que permitan adquirir y generar conocimiento, contribuyendo más allá de las ciencias a enriquecer y cualificar la formación ciudadana (García y Suárez, 2015). Existen diferentes tipos de competencias investigativas, de acuerdo con Buendía, Zambrano e Insuasty (2018), las cuales se resumen a continuación en el Cuadro 1.

\section{Cuadro 1 \\ Competencias de investigación científica}

\begin{tabular}{|c|c|}
\hline $\begin{array}{l}\text { Competencias } \\
\text { investigativas }\end{array}$ & Concepto \\
\hline $\begin{array}{l}\text { Competencias para } \\
\text { preguntar }\end{array}$ & Habilidad para formular preguntas de investigación cualitativa y cuantitativa. \\
\hline $\begin{array}{l}\text { Competencias } \\
\text { observacionales }\end{array}$ & $\begin{array}{l}\text { Habilidad para realizar observación científica de forma más selectiva y con ello registrar } \\
\text { aspectos importantes. }\end{array}$ \\
\hline Competencias reflexivas & $\begin{array}{l}\text { Habilidad para evaluar y reflexionar sobre el propio trabajo, para solucionar problemas y } \\
\text { tomar decisiones. }\end{array}$ \\
\hline $\begin{array}{l}\text { Competencias } \\
\text { propositivas }\end{array}$ & $\begin{array}{l}\text { Habilidad para proponer soluciones a los problemas detectados, empleando conceptos y } \\
\text { métodos de investigación explicativos o críticos. }\end{array}$ \\
\hline $\begin{array}{l}\text { Competencias } \\
\text { tecnológicas }\end{array}$ & $\begin{array}{l}\text { Habilidad para la selección y manejo de técnicas de recolección de datos, utilización de } \\
\text { software para el análisis de datos y la exposición de resultados. }\end{array}$ \\
\hline $\begin{array}{l}\text { Competencias } \\
\text { interpersonales }\end{array}$ & $\begin{array}{l}\text { Habilidad para relacionarse armónicamente entre los miembros del equipo de trabajo } \\
\text { investigativo. }\end{array}$ \\
\hline Competencias cognitivas & $\begin{array}{l}\text { Habilidad para la compresión, análisis, evaluación y distinción de teorías, tendencias y } \\
\text { metodologías científicas para interpretarlas o desarrollar nuevos conocimientos. }\end{array}$ \\
\hline $\begin{array}{l}\text { Competencias } \\
\text { procedimentales }\end{array}$ & $\begin{array}{l}\text { Habilidad para el manejo de técnicas necesarias para llevar un orden secuencial en las } \\
\text { diferentes etapas del proceso de investigación. }\end{array}$ \\
\hline Competencias analíticas & $\begin{array}{l}\text { Habilidad para comprender en profundidad los datos obtenidos en la investigación e } \\
\text { identificar y elementos importantes, especialmente la investigación cualitativa. }\end{array}$ \\
\hline $\begin{array}{l}\text { Competencias } \\
\text { comunicativas }\end{array}$ & $\begin{array}{l}\text { Habilidad para la generación y difusión de conocimientos de forma oral o escrita, derivados } \\
\text { de la investigación. }\end{array}$ \\
\hline
\end{tabular}

Fuente: Elaboración propia, 2020. 


\subsection{Habilidades psicométricas}

De acuerdo con Aragón (2015), la psicometría comprende "el conjunto de métodos, técnicas y teorías implicados en la medición de variables psicológicas; estudia las propiedades métricas exigibles en las mediciones psicológicas y establece las bases para que éstas se realicen de manera adecuada" (p.55); siendo una disciplina que cumple con los criterios que debe tener una práctica científica, tales como: Intentar describir hechos como son, trascenderlos, analizarlos y teorizarlos; se basa en el método científico, es clara y precisa, explicativa, verificable, predictiva y útil para la sociedad (Bunge, 2014); en otras palabras, la psicometría es una disciplina dedicada a la medición y evaluación cuantitativa de las capacidades así como características (actitudes, habilidades, emociones, creencias, conductas, entre otras) humanas, empleando instrumentos fidedignos para identificarlas, comprobarlas y predecirlas.

En este sentido, las habilidades psicométricas corresponden a aquellas destrezas que posee un investigador para la medición y evaluación de variables psicológicas a través del empleo de métodos e instrumentos, que permitan cuantificar dichas variables para analizarlas, comprobarlas y predecirlas. Estas habilidades, no solo están relacionadas con las competencias para aplicar instrumentos de medición y evaluación psicológica, sino también para analizar los resultados obtenidos de la aplicación de estos, en términos de análisis estadístico psicométrico, además de comprender, analizar y explicar la validez y confiablidad de dichos instrumentos.

\section{Metodología}

Se construyó un proyecto de aula basado en el aprendizaje cooperativo para el desarrollo de competencias investigativas relacionadas con la evaluación y medición psicológica, aplicado en cuatro grupos de clases de fundamentos de medición y evaluación psicológica de un programa de psicología de una institución de educación superior, con el propósito de establecer la confiabilidad y validez de los instrumentos utilizados para medir las variables: Comportamiento organizacional ciudadano, autonomía, clima escolar y habilidades sociales, a partir del análisis estadístico de las bases de datos derivadas de la aplicación previa de estos instrumentos.

\subsection{Procedimiento}

El proyecto de aula se llevó a cabo a lo largo de 3 fases, durante las cuales los estudiantes de la asignatura se apropiaron de las bases teóricas y prácticas, con la finalidad de realizar las actividades propuestas para la ejecución del proyecto y recibían asesorías externas del docente, para colocar en práctica dichos conocimientos y habilidades adquiridas durante el proceso de formación. En una primera fase, se constituyeron los equipos de trabajo conformados por cinco estudiantes por equipo y se distribuyeron los temas relacionados con las variables evaluadas, así como el nombre del instrumento utilizado para la evaluación de estas, con el propósito de que se hiciera una búsqueda bibliográfica inicial, relacionada con el tema para entender las variables y las características psicométricas del instrumento.

Luego de la búsqueda bibliográfica, se siguió a una segunda fase, que consistió en redactar un artículo científico con el objetivo de describir las características psicometrías del instrumento dado, a partir de una guía y orientación proporcionada por el docente con los elementos que debía contener dicho escrito. Finalmente, en una tercera fase, se capacitó a los estudiantes en el uso del programa estadístico SPSS para el análisis de las bases de datos resultantes de la aplicación previa de los instrumentos, con el fin de llevar a la práctica estos conocimientos para determinar la validez y confiablidad de estas pruebas, y posteriormente, hacer una presentación física y oral de los resultados así como las 
conclusiones derivadas de dicho análisis.

\section{Resultados y discusión}

A partir de las observaciones realizadas por el docente y el registro de las asesorías externas, se pudo evidenciar a lo largo del proyecto el interés y el compromiso de los estudiantes para apropiarse de los conocimientos, trabajar en equipo y asistir a las asesorías, así como la disposición para aprender a utilizar el programa estadístico SPSS durante la clase y en las visitas al docente, para orientación adicional con respecto al uso del mismo y el manejo de conceptos relacionados con validez y confiablidad de los instrumentos de evaluación designados; de la misma manera, se vio reflejada la apropiación teórica sobre las variables que evaluaban estos instrumentos, derivada de la búsqueda previa de información sobre cada temática en base datos institucionales y la entrega de un artículo científico al respecto, que incluía las características psicométricas de cada test.
En cuanto a la presentación oral de los resultados obtenidos del análisis estadístico de las bases de datos de la aplicación previa de estos instrumentos, se evidenció durante la exposición, una adecuada apropiación y uso de la terminología relacionada con evaluación y medición psicológica, puntalmente en lo concerniente a la validez y confiablidad de los instrumentos que fueron evaluados a través del programa estadístico; así mismo, se notó un buen trabajo en equipo y compenetración a la hora de socializar los resultados y expresar sus conclusiones con respecto a estos, evidenciando apoyo y motivación entre los compañeros durante los aportes individuales de cada miembro del equipo, mostrando interés para lograr el éxito de la tarea en conjunto.

Cuantitativamente, los cuatro grupos de clase evaluados obtuvieron un promedio de calificación de 4,0 al final de la presentación oral, con base en una escala de calificación de 0,0 a 5,0 , donde 0,0 es la menor puntuación y 5,0 corresponde a la mayor puntuación. Los promedios por grupos se presentan en la Tabla 1.

Tabla 1

\section{Promedio de calificación por grupos al final del curso}

\begin{tabular}{ccc}
\hline \multirow{2}{*}{ Grupos de clases } & \multicolumn{2}{c}{ Calificación final } \\
& Media & DE \\
\hline AN & 3,98 & 0,96 \\
AD & 4,42 & 0,71 \\
BD & 3,99 & 0,79 \\
CD & 3,59 & 1,15 \\
Total & $\mathbf{4 , 0 0}$ & $\mathbf{0 , 1 9}$ \\
\hline
\end{tabular}

Nota: DE: Desviación estándar. La escala de calificación es 0,0 a 5,0, donde 0,0 es la menor puntuación y 5.0 la mayor puntuación.

Fuente: Elaboración propia, 2020.

Estos resultados evidencian la efectividad del aprendizaje cooperativo en la adquisición de competencias investigativas relacionadas con habilidades psicométricas, teniendo en cuenta que el trabajo en los equipos permitió que los estudiantes se apropiaran no lo solo de los conocimientos teóricos en materia de investigación, sino que también pudieran llevarlos a la práctica a través de la búsqueda bibliográfica, la redacción del 
artículo científico, el empleo del programa estadístico SPSS y el posterior análisis y conclusión de los resultados derivados del uso de este recurso tecnólogo para obtener la confiablidad y validez de los instrumentos, que era el propósito central del proyecto de aula implementado como parte de la estrategia pedagógica del docente a cargo.

Considerando los aspectos anteriores, es posible afirmar que este tipo de estrategia permitió un porcentaje de aprendizaje del $75 \%$ según la pirámide del aprendizaje de Cody Blair, teniendo en cuenta que se realizaron prácticas durante el curso para afianzar mejor los conocimientos teóricos tratados, lo cual favoreció al desarrollo de diferentes competencias investigativas, como la capacidad para formular preguntas de investigación (competencias para preguntar), evaluar y reflexionar sobre el propio trabajo individual $y$ en equipo (competencias reflexivas e interpersonales), proponer soluciones usando conceptos y métodos de investigación explicativos o críticos (competencias propositivas).

Además de ello, emplear programas informáticos para el análisis estadístico de datos (competencias tecnológicas), comprender, evaluar y distinguir teorías y metodologías científicas para interpretarlas, desarrollar nuevos saberes o analizar información obtenida de la investigación (competencias cognitivas y analíticas); manejo de técnicas para llevar a cabo un proceso de investigación por etapas (competencias procedimentales); y finalmente, comunicar y construir el conocimiento científico de forma oral o escrita (competencias comunicativas).

En este sentido, se muestra que el aprendizaje cooperativo incidió poderosamente en el desarrollo de competencias investigativas psicométricas, así como en otros aspectos vinculados a la formación académica y profesional, teniendo en cuenta que este tipo de aprendizaje afecta positivamente el logro estudiantil, la motivación para el aprendizaje, las relaciones intergrupales, el pensamiento crítico y creativo y la resolución de problemas, así como otra serie de recursos
(Baloche y Brody, 2017; Compte y Sánchez, 2019); aumenta la confianza y el trabajo en equipo eficiente (León-del-Barco, et al., 2017; Hebles, Yaniz-Álvarez-de-Eulate y Jara, 2019); y, permite el desarrollo de habilidades investigativas, sobre todo cuando se incluyen asesorías o capacitaciones en investigación, puesto que estas mejoran el conocimiento y las habilidades de investigación cuando se llevan a cabo en términos del cumplimiento exitoso de tareas de evaluación (Al-Tannir, et al., 2018).

Existe un gran número de investigaciones científicas que confirman la efectividad del aprendizaje cooperativo, especialmente en el entorno de la educación superior, como se mencionó anteriormente. Algunos estudios demuestran que este tipo de aprendizaje es efectivo para fomentar diferentes estilos de trabajo, alentar la asistencia diaria a las clases y permitir el establecimiento de nuevos vínculos de relación entre estudiantes que no se conocían con anterioridad durante el trabajo en equipo (Martín-Salinas y Cid-Galán, 2018).

También se ha encontrado, que el aprendizaje cooperativo se asocia con relaciones más positivas entre el desafío académico, la interacción cooperativa, las ganancias de aprendizaje y la satisfacción general de estudiantes universitarios (Tadesse, Gillies y Manathunga, 2020). Otras investigaciones, han evidenciado que estrategias pedagógicas basadas en el aprendizaje cooperativo o específicamente en el desarrollo de competencias investigativas mejoran las habilidades para la investigación y la escritura académica (Prahmana, 2017; Sutarman, Sunendar y Mulyati, 2019; Lander, Seeho y Foster, 2019).

Finalmente, en la actualidad existe evidencia científica que indica que el desarrollo de habilidades de investigación en los estudiantes, es de utilidad para conectar con el mercado laboral actual o futuro, debido a que pueden ser competencias requeridas para trabajar dentro de una organización o de manera autónoma e independiente (Willison, Sabir y Thomas, 2017); considerando esto, Maddens, et al. (2020) enfatizan en la 
Hernández Sánchez, Irmina; Lay Nelson; Herrera Henry y Rodríguez, Marcela

Estrategias pedagógicas para el aprendizaje y desarrollo de competencias investigativas en

estudiantes universitarios

importancia de las características curriculares para fomentar las habilidades de investigación de los estudiantes, así pues se recomienda incluir en los programas de formación profesional estrategias pedagógicas basadas en el desarrollo de este tipo de competencias.

\section{Conclusiones}

Los resultados obtenidos en esta experiencia de formación académica permiten concluir que estrategias pedagógicas como proyectos de aula, basados en el aprendizaje cooperativo, son efectivos para la adquisición y desarrollo de diferentes tipos de competencias investigativas, tales como búsqueda de información científica, redacción de artículos científicos, análisis y procesamiento de datos, manejo de recursos y metodologías científicas procedimentales e informáticas, obtención de características psicométricas como la validez y confiablidad de instrumentos de medición, entre otras, relacionadas con habilidades de medición y evaluación psicológica, logrando un porcentaje significativo en la adquisición y aplicación de este tipo de competencias en el contexto universitario.

Por otra parte, fomenta un mejor aprovechamiento y uso de los conocimientos adquiridos en la academia, potencia el trabajo en equipo, las relaciones sociales y el apoyo mutuo entre los estudiantes, motiva el proceso de aprendizaje y permite el logro de resultados académicos exitosos.

En este sentido, se recomienda aplicar este tipo de metodologías pedagógicas en el ámbito de la educación superior, con el fin de lograr la adquisición y aplicación efectiva de competencias de investigación científica, las cuales son sumamente importantes a la hora de acceder a oportunidades en el mercado laboral, considerando su utilidad para llevar a cabo diferentes tareas donde se requieran, teniendo en cuenta que las exigencias actuales de esta sociedad globalizada traen consigo nuevos retos y a su vez demandan respuestas oportunas, válidas y confiables, bajo un criterio científico que favorezca a una mejor solución de los problemas que puedan presentarse en los diferentes ámbitos laborales.

\section{Referencias bibliográficas}

Al-Tannir, M., Abu-Shaheen, A., Alsumaih, S., AlMukaibil, N. F., AlHarbi, R., Heena, H., Sallout, L., Mahha, A., Mohammed, N., y AlFayyad, I. (2018). Research knowledge and skills among medical and allied health students attending a summer research course: A pretest and posttest analysis. Cureus, 10(8), e3132. https://doi.org/10.7759/ cureus. 3132

Ambrose, S. A., Bridges, M. W., Lovett, M. C., Norman, M. K., y Diprieto, M. (2017). Cómo funciona el aprendizaje. 7 principios basados en la investigación para una enseñanza inteligente. Editorial Universidad del Norte.

Aragón, L. E. (2015). Evaluación psicológica. Historia, fundamentos teóricoconceptuales y psicometría. Manual Moderno.

Baloche, L., y Brody, C. M. (2017). Cooperative learning: exploring challenges, crafting innovations. Journal of Education for Teaching, 43(3), 274-283. https://doi.org/10.108 $\underline{0 / 02607476.2017 .1319513}$

Barreiro, P. E. (2015). Las competencias investigativas del estudiante en el proceso de enseñanza aprendizaje. Revista Electrónica Formación y Calidad Educativa (REFCalE), 3(1), 57-68.

Buendía, X. P., Zambrano, L. C., e Insuasty, E. A. (2018). El desarrollo de competencias investigativas de los docentes en formación en el contexto de la práctica pedagógica. FOLIOS, (47), 179-195. https://doi. org/10.17227/folios.47-7405 
Bunge M. (2014). La ciencia: Su método y su filosofía. Penguin Random House.

Castillo, V., Yahuita, J., y Garabito, R. (2006). Estrategias docentes para un aprendizaje significativo. Cuadernos del Hospital de Clínicas, 51(1), 96101.

Cejas, M. F., Rueda, M. J., Cayo, L. E., y Villa, L. C. (2019). Formación por competencias: Reto de la educación superior. Revista de Ciencias Sociales (Ve) $X X V(1), 94-101$.

Cifuentes, P., y Meseguer, P. (2015). Trabajo en equipo frente a trabajo individual. Ventajas del aprendizaje cooperativo en el aula de traducción. Tonos Digital: Revista de Estudios Filológicos, (28), $1-21$.

Coaquira, C. (2020). Prácticas pedagógicas desde el enfoque socioformativo: Una autoevaluación docente en Perú. Revista de Ciencias Sociales (Ve), XXVI(E-2), 260-274. https://doi. org/10.31876/rcs.v26i0.34126

Compte, M., y Sánchez, M. (2019). Aprendizaje colaborativo en el sistema de educación superior ecuatoriano. Revista de Ciencias Sociales (Ve), $X X V(2), 131-140$.

Dejo-Oricain, N. (2015). Adquisición de competencias en el marco del aprendizaje cooperativo: valoración de los estudiantes. REDU. Revista de Docencia Universitaria, 13(1), 339-359. $\quad$ https://doi.org/10.4995/ redu. 2015.6434

Esguerra, G., y Guerrero, P. (2010). Estilos de aprendizaje y rendimiento académico en estudiantes de psicología. Revista Diversitas: Perspectivas en Psicología, 6(1), 97-109.

Gamboa, M. C., García, Y., y Beltrán, M. (2013). Estrategias pedagógicas y didácticas para el desarrollo de las inteligencias múltiples y el aprendizaje autónomo. Revista de Investigaciones UNAD, 12(1), 101-128. https://doi. org/10.22490/25391887.1162

Garcés, J., y Mora, C. (2020). Estrategias de aprendizaje para mitigar la deserción estudiantil en el marco de la COVID-19. SUMMA. Revista Disciplinaria en Ciencias Económicas y Sociales, 2(E), 49-55.

García, F. S., Tejeda, R., y Torres, R. M. (2014). La formación de competencias científico investigativas para la sostenibilidad ambiental en el ingeniero agropecuario. Revista Electrónica Formación y Calidad Educativa (REFCalE), 2(3), 59-70.

García, F. S., y Suárez, C. (2015). La formación de competencias científicas investigativas en la carrera de Ingeniería Agropecuaria de la Universidad Laica "Eloy Alfaro" de Manabí, campus Pedernales. Revista Universidad y Sociedad, 7(2), 115120.

Gómez-Ejerique, C., y López-Cantos, F. (2019). Application of innovative teaching-learning methodologies in the classroom. Coaching, flippedclassroom and gamification. A case study of success. Multidiscilinary Journal for Education, Social and Technological Sciences, 6(1), 46-70. $\quad$ https://doi.org/10.4995/ muse.2019.9959

González, N., García, R., y Ramírez, A. (2015). Aprendizaje cooperativo y tutoría entre iguales en entornos virtuales universitarios. Estudios Pedagógicos, $X \operatorname{LI}(1), 111-124$.

Hebles, M., Yaniz-Álvarez-de-Eulate, C., y Jara, M. (2019). Impact of cooperative learning on teamwork competence. Academia Revista Latinoamericana de Administración, 32(1), 93-106. https:// doi.org/10.1108/ARLA-10-2018-0217 
Hernández Sánchez, Irmina; Lay Nelson; Herrera Henry y Rodriguez, Marcela Estrategias pedagógicas para el aprendizaje y desarrollo de competencias investigativas en estudiantes universitarios

Lander, J., Seeho, S., y Foster, K. (2019). Learning practical research skills using. An academic paper framework - an innovative, integrated approach. Health Professions Education, 5(2), 136-145. https://doi.org/10.1016/j. hpe.2018.06.002

Lay N., Ramírez, J., y Parra, M. (2019). Desarrollo de conductas ciudadanas en estudiantes del octavo grado de una institución educativa de Barranquilla. En R. López, Memorias del I Congreso Internacional en Educación e Innovación en Educación Superior. Caracas, Venezuela. Fondo Editorial UPEL.

León-del-Barco, B., Mendo, S., FelipeCastaño, E., Polo-del-Rio, M. I., y Fajardo-Bullón, F. (2017). Potencia de equipo y aprendizaje cooperativo en el ámbito universitario. Revista de Psicodidáctica, 22(1), 9-15. https://doi. org/10.1387/RevPsicodidact.14213

López, G., y Acuña, S. (2015). Aprendizaje cooperativo en el aula. Inventio. La Génesis de la Cultura Universitaria en Morelos, 7(14), 28-37.

Lucumi, P., y González, M. A. (2015). El ambiente digital en la comunicación, la actitud y las estrategias pedagógicas utilizadas por docentes. TED: Tecné Episteme y Didaxis, (37), 109-129. https://doi.org/10.17227/01213814.37 $\underline{\text { ted109.129 }}$

Maddens, L., Depaepe, F., Janssen, R., Raes, A., y Elen, J. (2020). Research skills in upper secondary education and in first year of university. Educational Studies, 3(5), 1-17. https://doi.org/10. $\underline{1080 / 03055698.2020 .1715204}$

Martínez, P., Martínez, M., y MuñozCantero, J-M. (2008). Aprendizaje de competencias en educación superior. Revista Galego-Portuguesa de Psicoloxía e Educación, 16, 195-215.
Martínez-Garcés, J., y Garcés-Fuenmayor, J. (2020). Competencias digitales docentes y el reto de la educación virtual derivado de la covid-19. Educación y Humanismo, 22(39), 1-16. https://doi.org/10.17081/ eduhum.22.39.4114

Martín-Salinas, C., y Cid-Galán, M. L. (2018). Experiencia de aprendizaje cooperativo en una asignatura optativa del Grado en Enfermería. Educación Médica, 19(5), 288-293. https://doi. org/10.1016/j.edumed.2017.10.035

Naciones Unidas (2018). La Agenda 2030 y los Objetivos de Desarrollo Sostenible: Una oportunidad para América Latina y el Caribe. Objetivos, metas e indicadores mundiales. CEPAL. https://www.cepal.org/es/ publicaciones/40155-la-agenda2030-objetivos-desarrollo-sostenibleoportunidad-america-latina-caribe

Niebles, W., Martínez-Bustos, P. y NieblesNúñez, L. (2020). Competencias matemáticas como factor de éxito en la prueba pro en universidades de Barranquilla, Colombia. Educación y Humanismo, 22(38), 1-16. https://doi. org/10.17081/eduhum.22.38.3590

Organización para la Cooperación y el Desarrollo Económico - OCDE (2006). Programa para la Evaluación Internacional de Alumnos PISA (2006). Marco de la evaluación. Conocimientos y habilidades en Ciencias, Matemáticas y Lectura. https://www.oecd.org/pisa/39732471. pdf

Parra, M. A., Hernández-Sánchez, I., Maussa, E., y Guerrero, M. B. (2018). Elementos que definen una estrategia pedagógica en la escuela de padres del ICBF del sur-occidente de Barranquilla. Hexágono Pedagógico, 9(1), 1-14.

Pirela, A., González, N., Pérez, R., y Carrillo, 
J. (2019). Formación en competencias investigativas en los estudiantes de una universidad inclusiva. SUMMA. Revista Disciplinaria en Ciencias Económicas y Sociales, 1(1), 35-51.

Prahmana, R. C. I. (2017). The role of researchbased learning to enhance students' research and academic writing skills. Journal of Education and Learning (EduLearn), 11(3), 351-366.

Rabanal, R., Huamán, C. R., Murga, N. L., y Chauca, P. (2020). Desarrollo de competencias personales y sociales para la inserción laboral de egresados universitarios. Revista de Ciencias Sociales (Ve), XXVI(2), 250$258 . \quad$ https://doi.org/10.31876/rcs. v26i2.32438

Rosario, Y., y Ferrer, E. A. (2014). Estrategia para la formación de competencias investigativas en estudiantes de la carrera ingeniería informática. Didáctica y Educación, V(4), 143-162.

Rubio, M. J., Torrado, M., Quirós, C., y Valls, R. (2018). Autopercepción de las competencias investigativas en estudiantes de último curso de pedagogía de la universidad de Barcelona para desarrollar su trabajo de fin de grado. Revista Complutense de Educación, 29(2), 335-354. https:// doi.org/10.5209/RCED.52443

Sutarman, Sunendar, D., y Mulyati, Y. (2019). Investigating cooperative learning model based on interpersonal intelligence on language learner's skill to write article. International Journal of Instruction, 12(4), 201-218. https:// doi.org/10.29333/iji.2019.12413a

Tadesse, T., Gillies, R. M., y Manathunga, C. (2020). Shifting the instructional paradigm in higher education classrooms in Ethiopia: What happens when we use cooperative learning pedagogies more seriously? International Journal of Educational Research, 99, 101509. https://doi. org/10.1016/j.ijer.2019.101509

Valbuena, A. (2017). Yo no quiero ir a esta escuela: Los pecados de nuestro sistema educativo. Finis Terrae Ediciones.

Vallet-Bellmunt, T., Rivera-Torres, P., ValletBellmunt, I., y Vallet-Bellmunt, A. (2017). Aprendizaje cooperativo, aprendizaje percibido y rendimiento académico de la enseñanza de marketing. Educación XX1, 20(1), 277-297. https://doi.org/10.5944/ educxx1.17512

Vargas, F., Casanova, F., y Montanaro, L. (2001). El enfoque de la competencia laboral: Manual de formación. OIT/ Cinterfor.

Willison, J., Sabir, F., y Thomas, J. (2017). Shifting dimensions of autonomy in students research and employment. Higher Education Research and Development, 36(2), 430-443. https:// doi.org/10.1080/07294360.2016.1178 $\underline{216}$ 